\title{
Invariants for Time-Dependent Harmonic Oscillators from the Real Representation of Solution
}

\author{
M.-L. LIANG ${ }^{a, b}$, Z.-G. ZHANG ${ }^{a}$ AND B. YUAN ${ }^{a}$ \\ ${ }^{a}$ Department of Applied Physics, School of Science \\ Tianjin University, Tianjin 300072, China \\ ${ }^{b}$ LiuHui Center for Applied Mathematics, Tianjin 300071, China
}

(Received October 13, 2003)

\begin{abstract}
The invariants for the time-dependent one-dimensional harmonic oscillator and the time-dependent two-dimensional harmonic oscillator in a static magnetic field are derived from the real representation of the exact solution to the equation of motion. Mathematically, the orthogonal functions invariant is the angular momentum of an isotropic time-dependent two-dimensional harmonic oscillator. Based on the invariants obtained here, the wave function for time-dependent two-dimensional harmonic oscillator in a static magnetic field in cylindrical coordinate is simply derived and the dynamical and geometrical phases are easily got by expressing the wave function as the superpositions of the wave functions of time-dependent two-dimensional harmonic oscillator in rectangular coordinate. For the driven system, the driving induced dynamical phase and the geometrical phase are respectively associated with the classical Hamiltonian and de Broglie wave of the center motion of the wave function.
\end{abstract}

PACS numbers: $45.20 . \mathrm{Jj}, 03.65 .-\mathrm{w}$

\section{Introduction}

The invariant for the time-dependent one-dimensional harmonic oscillator (TOHO) has been obtained by different methods [1-3]. Recently, for the harmonic oscillator with a unit mass, the authors of Ref. [4] presented an elementary derivation of the invariant using the complex representation of the solution. It is reasonable to expect that the method in [4] may apply to the physical harmonic oscillator with a real coordinate, but it does not. For the general solution in [4]: 


$$
q_{g}=A_{+} \mathrm{e}^{\mathrm{i} s_{+}}+\sigma A_{+} \mathrm{e}^{-\mathrm{i} s_{+}}
$$

there exists the invariant

$$
Q=A_{+}^{2}\left(1-\sigma^{2}\right) \frac{\mathrm{d} s_{+}}{\mathrm{d} t} .
$$

To have a real coordinate, the parameter $\sigma$ should be set to unit. If doing so, the invariant $Q$ will vanish and thus the derivation breaks up. Hence, for the physical harmonic oscillator with a real coordinate the method in [4] is ineffective. In another words, the authors actually discussed a complex oscillator.

Considering that physically the coordinate of the harmonic oscillator is real, it is necessary to give a re-derivation of the invariant from the real representation of the solution. In our treatment, the mass is not unity and allowed to change with time [5]. Besides TOHO, we also deal with time-dependent two-dimensional harmonic oscillator (TTHO) in a static magnetic field [6]. The dynamical and geometrical phases [7,8], properties of the driven systems are all discussed.

This article is organized as follows. The next and third sections deal with TOHO and TTHO in a static magnetic field, respectively. The fourth section is a simple re-derivation of the wave function for TTHO in a static magnetic field based on the invariants found in Sec. 3. The dynamical and geometrical phases are also explicitly given. Discussion of the driven system is in Sec. 5. The conclusion is in Sec. 6.

\section{The one-dimensional harmonic oscillator}

The TOHO with time-dependent mass and frequency is described by the equation of motion

$$
\frac{\mathrm{d}}{\mathrm{d} t}[M(t) \dot{q}]+M(t) \omega^{2}(t) q=0,
$$

where $M(t)$ and $\omega(t)$ are the time-dependent mass and frequency. The dot on the physical quantity means time derivative. In the time-independent case, Eq. (3) has the well-known solution

$$
q(t)=A_{0} \cos \left(\omega t+\theta_{0}\right)
$$

with $A_{0}$ and $\theta_{0}$ being two constants. When the mass and frequency are time-dependent, the solution may be considered as the generalization of (4)

$$
q(t)=A(t) \cos f(t)
$$

where $f(t)$ and $A(t)$ are two functions of time to be found. Physically, $f(t)$ is the classical total phase. Substituting (5) into (3) and setting the coefficients before $\cos f(t)$ and $\sin f(t)$ zero, the equations for the two unknown functions are got

$$
\begin{aligned}
& M A \ddot{f}+2 M \dot{A} \dot{f}+\dot{M} A \dot{f}=0, \\
& M \ddot{A}-M A \dot{f}^{2}+\dot{M} \dot{A}+M \omega^{2} A=0 .
\end{aligned}
$$


Equation (6a) can be rewritten as

$$
\frac{\mathrm{d}}{\mathrm{d} t}\left(M A^{2} \frac{\mathrm{d} f}{\mathrm{~d} t}\right)=0,
$$

which agrees with that in [4] when $M=1$. From (7), we know that there exists an invariant

$$
Q=M A^{2} \frac{\mathrm{d} f}{\mathrm{~d} t} .
$$

If making

$$
A=\rho(t) \sqrt{Q}
$$

Eq. (8) will admit the solution

$$
f(t)=\int_{0}^{t} \frac{\mathrm{d} \tau}{M(\tau) \rho^{2}(\tau)}+\theta_{0}
$$

The classical total phase $f(t)$ is derived from the exact solution to the classical equation of motion automatically. Substituting (10) into (6b), the auxiliary equation for $\rho(t)$ is found

$$
\ddot{\rho}+\frac{\dot{M} \dot{\rho}}{M}+\omega^{2} \rho=\frac{1}{M^{2} \rho^{3}},
$$

which is consistent with that in [5]. From (5) and (9), we have

$$
\cos f(t)=q(t) / A(t)=q(t) /(\rho \sqrt{Q}) .
$$

Doing time derivative on the two sides, one obtains

$$
\sin f(t)=(M \dot{q} \rho-M \dot{\rho} q) / \sqrt{Q} .
$$

Replacing $\dot{q}$ by the momentum $p=M \dot{q}$, from the basic mathematical equality $\cos ^{2} f(t)+\sin ^{2} f(t)=1$, we get

$$
Q=\left(\frac{q}{\rho}\right)^{2}+(\rho p-M \dot{\rho} q)^{2}
$$

$Q / 2=I$ is just the Lewis-Riesenfeld invariant for TOHO [5]. The coordinate can be finally written as $q(t)=\sqrt{Q} \rho(t) \cos f(t)$. Clearly, the invariant affects the amplitude.

\section{On the orthogonal functions invariant}

Let us suppose that $q_{1}$ and $q_{2}$ are two solutions to Eq. (1):

$$
\begin{aligned}
& \frac{\mathrm{d}}{\mathrm{d} t}\left[M(t) \dot{q}_{1}\right]+M(t) \omega^{2}(t) q_{1}=0 \\
& \frac{\mathrm{d}}{\mathrm{d} t}\left[M(t) \dot{q}_{2}\right]+M(t) \omega^{2}(t) q_{2}=0
\end{aligned}
$$


Multiplying (15b) by $q_{1}$ minus (15a) multiplied by $q_{2}$ gives $\mathrm{d}\left(q_{1} M \dot{q}_{2}-\right.$ $\left.q_{2} M \dot{q}_{1}\right) / \mathrm{d} t=0$, which results in an invariant

$$
Q_{12}=q_{1} p_{2}-q_{2} p_{1}
$$

with $p_{1}=M \dot{q}_{1}$ and $p_{2}=M \dot{q}_{2}$ being the momenta corresponding to the solutions $q_{1}$ and $q_{2}$. Mathematically, $(15 \mathrm{a}, \mathrm{b})$ describes an isotropic TTHO and $Q_{12}$ is the angular momentum. From this point of view, we may physically understand why "the only non-vanishing contribution to the invariant $Q_{12}$ comes from functions that are $90^{\circ}$ out of phase" [4]. As is known, the angular momentum will be zero if the two components $x=q_{1}$ and $y=q_{2}$ of a two-dimensional oscillator are in phase.

Next we consider the TTHO in a static magnetic field [6]. The Hamiltonian is $[6]$ :

$$
H=\frac{p_{x}^{2}+p_{y}^{2}}{2 M(t)}+\frac{1}{2} \varpi_{c} L_{z}+\frac{1}{2} M(t) \Omega(t)^{2}\left(x^{2}+y^{2}\right),
$$

where $p_{x}=M \dot{x}+e B_{0} y / 2$ and $p_{y}=M \dot{y}-e B_{0} y / 2$ are the canonical momenta, $L_{z}=x p_{y}-y p_{x}$ is the canonical angular momentum in the axial $z$ direction, $\varpi_{\mathrm{c}}=$ $e B_{0}(t) / M(t)$ is the cyclotronic frequency of oscillation. The general frequency $\Omega(t)$ takes the form $\Omega^{2}(t)=\varpi_{c}^{2}(t) / 4+\omega^{2}(t)$. The invariant in quantum mechanics is defined by $\frac{\mathrm{d} I}{\mathrm{~d} t}=\frac{\partial I}{\partial t}+\frac{1}{\mathrm{i} \hbar}[I, H]=0$. Using the following relations:

$$
\begin{aligned}
& {\left[x^{2}+y^{2}, L_{z}\right]=0, \quad\left[p_{x}^{2}+p_{y}^{2}, L_{z}\right]=0,} \\
& {\left[x p_{x}+p_{x} x+y p_{y}+p_{y} y, L_{z}\right]=0}
\end{aligned}
$$

we can prove that there exist two invariants: one is the canonical angular momentum $I_{1}=L_{z}$ and the other is

$$
\begin{aligned}
I_{2}= & \frac{1}{2}\left[\left(\frac{x}{g}\right)^{2}+\left(g p_{x}-M \dot{g} x\right)^{2}\right]+\frac{1}{2}\left[\left(\frac{y}{g}\right)^{2}+\left(g p_{y}-M \dot{g} y\right)^{2}\right] \\
& =\frac{g^{2}}{2}\left(p_{x}^{2}+p_{y}^{2}\right)+\frac{x^{2}+y^{2}}{2 g^{2}}-M g \dot{g}\left(x p_{x}+p_{x} x+y p_{y}+p_{y} y\right),
\end{aligned}
$$

where the function $g(t)$ satisfies the equation

$$
\ddot{g}+\frac{\dot{M} \dot{g}}{M}+\Omega^{2} g=\frac{1}{M^{2} g^{3}} .
$$

Next we derive the invariants from the exact solutions to the equations of motion.

The equations of motion for TTHO in a static magnetic field are [6]:

$$
\begin{aligned}
& \ddot{x}+\frac{\dot{M}}{M} \dot{x}+\omega^{2} x=-\varpi_{c} \dot{y}, \\
& \ddot{y}+\frac{\dot{M}}{M} \dot{y}+\omega^{2} y=\varpi_{c} \dot{x},
\end{aligned}
$$


which can be rewritten as

$$
\begin{aligned}
\frac{\mathrm{d}}{\mathrm{d} t}(M \dot{x})+M \omega^{2} x & =-e B_{0} \dot{y}, \\
\frac{\mathrm{d}}{\mathrm{d} t}(M \dot{y})+M \omega^{2} y & =-e B_{0} \dot{x} .
\end{aligned}
$$

Substituting $x=r(t) \cos \phi(t), y=r(t) \sin \phi(t)$ into any of the equations in (22), we have

$$
\begin{aligned}
& \ddot{r}-r \dot{\phi}^{2}+\frac{\dot{M}}{M} \dot{r}+\omega^{2} r=-\frac{e B_{0}}{M} r \dot{\phi}, \\
& r \ddot{\phi}+2 \dot{r} \dot{\phi}+\frac{\dot{M}}{M} r \dot{\phi}=\frac{e B_{0}}{M} \dot{r} .
\end{aligned}
$$

By some inspection, we notice that the second equation has the solution $\dot{\phi}=e B_{0} /(2 M)$ or

$$
\phi(t)=\int_{0}^{t} \frac{\varpi_{c}(\tau)}{2} d \tau
$$

Substituting (24) into the first equation of (23), we obtain

$$
\frac{\mathrm{d}}{\mathrm{d} t}(M \dot{r})+M \Omega^{2} r=0
$$

which is a TOHO and has the solution

$$
r=\sqrt{Q} g(t) \cos G(t), \quad G(t)=\int_{0}^{t} \frac{1}{M(\tau) g^{2}(\tau)} \mathrm{d} \tau+\theta_{0} .
$$

The functions $G(t)$ and $\phi(t)$ are the classical phases. From the quantum phase $\alpha_{n, m}(t)$ in [6], one can get the quantum-classical relation $\alpha_{n, m}(t)=-(2 n+$ $|m|+1) G(t)-m \phi(t)$ for $\theta_{0}=0$, with $n$ and $m$ being the radial and magnetic quantum numbers, respectively. Using (26), the coordinates $x$ and $y$ now take the form

$$
\begin{aligned}
& x=\sqrt{Q} g(t) \cos G(t) \cos \phi(t), \\
& y=\sqrt{Q} g(t) \cos G(t) \sin \phi(t) .
\end{aligned}
$$

Straightforward calculations give

$$
Q \cos ^{2} G(t)=\frac{x^{2}+y^{2}}{g^{2}}
$$

From (27), we can also get

$$
Q \sin ^{2} G(t)=\left(g p_{x}-M \dot{g} x\right)^{2}+\left(g p_{y}-M \dot{g} y\right)^{2} .
$$

Combining (28) and (29) yields the invariant $I_{2}=Q / 2$.

The invariant $I_{1}$ or the canonical momentum can be achieved from Eqs. (22) directly. Multiplying the first equation by $y$ minus the second equation multiplied by $x$ results in

$$
\frac{\mathrm{d}}{\mathrm{d} t}\left[y\left(M \dot{x}+\frac{e B_{0}}{2} y\right)-x\left(M \dot{y}-\frac{e B_{0}}{2} x\right)\right]=0,
$$

which produces an invariant $x p_{y}-y p_{x}=L_{z}=I_{1}$. 


\section{Dynamical and geometrical phases of TTHO in a magnetic field}

From the invariants $I_{1}$ and $I_{2}$, the wave function of TTHO in a static magnetic field can be obtained by standard method $[1,7,8]$. In cylindrical coordinate, the eigenfunction of $I_{1}$ is

$$
\phi_{m}(\varphi)=\frac{1}{\sqrt{2 \pi}} \exp (\mathrm{i} m \varphi), \quad m=0, \pm 1, \pm 2, \ldots
$$

The invariant $I_{2}$ in (19) may be rewritten in the form

$$
I_{2}=\frac{1}{2}\left[\left(\frac{r}{g}\right)^{2}+g^{2}\left(p-M \frac{\dot{g}}{g} r\right)^{2}\right]
$$

which agrees with the Hamiltonian of a two-dimensional harmonic oscillator in a magnetic field with a vector potential $a=m \dot{g} r / g$. By an unitary transformation

$$
U=\exp \left(\mathrm{i} \frac{M \dot{g}}{2 \hbar g} r^{2}\right)
$$

the invariant $I_{2}$ changes into

$$
I_{2}^{\prime}=U^{+} I_{2} U=\frac{1}{2}\left[\left(\frac{r}{g}\right)^{2}+g^{2} p^{2}\right]
$$

which is a two-dimensional harmonic oscillator with mass $1 / g^{2}$ and a unit frequency. The eigenstates can be found in textbooks on quantum mechanics. The angular part of the eigenfunction of $I_{2}^{\prime}$ or $I_{2}$ is also (31). Following the standard process $[7,8]$, straightforward calculations can yield the wave function.

Instead of using the invariant theory $[1,7,8]$, we can also obtain the wave function of TTHO in a static magnetic field by the simple and elementary trial function method $[9,10]$. As $\phi_{m}(\varphi)$ is the eigenfunction of $I_{1}$ or the angular part of the eigenfunction of $I_{2}$, from the invariant theory $[1,7,8]$ the wave function can be written as

$$
\psi(r, t)=\phi(r, t) \phi_{m}(\varphi) .
$$

Substituting (35) into the Schrödinger equation $H \psi(r, t)=i \hbar \partial \psi(r, t) / \partial t$ and noticing that the Hamiltonian in cylindrical coordinate is

$$
H=-\frac{\hbar^{2}}{2 M(t) r} \frac{\partial}{\partial r}\left(r \frac{\partial}{\partial r}\right)+\frac{L_{z}^{2}}{2 M(t) r^{2}}+\frac{1}{2} M(t) \Omega^{2}(t) r^{2}+\frac{1}{2} \varpi_{c} L_{z},
$$

we have

$$
H_{\mathrm{eff}} \phi(r, t)=\mathrm{i} \hbar \frac{\partial \phi(r, t)}{\partial t}
$$

where

$$
H_{\mathrm{eff}}=-\frac{\hbar^{2}}{2 M(t) r} \frac{\partial}{\partial r}\left(r \frac{\partial}{\partial r}\right)+\frac{m^{2} \hbar^{2}}{2 M(t) r}+\frac{1}{2} M(t) \Omega^{2}(t) r^{2}+\frac{1}{2} \varpi_{\mathrm{c}} m \hbar .
$$


To solve Eq. (37), we assume

$$
\phi(r, t)=\mathrm{e}^{\mathrm{i} f(r, t)} \frac{\phi_{N m}(R)}{g(t) \sqrt{\hbar}}, \quad R=\frac{r}{g(t) \sqrt{\hbar}},
$$

where $g(t)$ and $f(r, t)$ are two functions to be found. (In fact, the function is that in (20).) The function $\phi_{N m}(R)$ satisfies the equation

$$
\left[-\frac{1}{2 R} \frac{\partial}{\partial R}\left(R \frac{\partial}{\partial R}\right)+\frac{m^{2}}{2 R^{2}}+\frac{1}{2} R^{2}\right] \phi_{N m}(R)=(N+1) \phi_{N m}(R),
$$

which is the radial equation of a two-dimensional harmonic oscillator with unit mass, frequency and Planck constant. The quantum number $N=2 n+|m|$ is the main number [11]. (Here, $n$ is the radial quantum number.) Substituting (39) into (37), we derive the following equations:

$$
\begin{aligned}
& \frac{\hbar}{M(t)} \frac{\partial f(r, t)}{\partial r}=\frac{\dot{g}}{g} r \\
& -\hbar \frac{\partial f(r, t)}{\partial t}=(N+1) \frac{\hbar}{M(t) g^{2}}+\frac{\hbar^{2}}{2 M(t)}\left(\frac{\partial f}{\partial r}\right)^{2}-\frac{1}{2 M g^{4}} r^{2} \\
& \quad+\frac{1}{2} M \Omega^{2} r^{2}+\frac{1}{2} \varpi_{c} m \hbar .
\end{aligned}
$$

Consistency of the two equations demands $\partial^{2} f / \partial r \partial t=\partial^{2} f / \partial t \partial r$, which results in the condition (20). The first equation of (41) admits the solution

$$
f(r, t)=\frac{M \dot{g}}{2 h g} r^{2}+\gamma(t)
$$

Inserting (42) into the second equation of (41), the function $\gamma(t)$ is found

$$
\gamma(t)=-(N+1) \int_{0}^{t} \frac{\mathrm{d} \tau}{M(\tau) g^{2}(\tau)}-\frac{m}{2} \int_{0}^{t} \varpi_{c}(\tau) \mathrm{d} \tau
$$

which agrees with that in [6]. Up to now the wave function (35) is got

$$
\begin{aligned}
& \psi(r, t)=\phi_{N m}(r, \varphi, t) \exp \left[i \gamma_{N m}(t)\right] \\
& \phi_{N m}(r, \varphi, t)=\phi_{N m}(R) \phi_{m}(\varphi) \exp \left(\frac{\mathrm{i} M \dot{g}}{2 \hbar g} r^{2}\right),
\end{aligned}
$$

where the quantum phase is

$$
\gamma_{N m}(t)=\gamma(t)
$$

The dynamical and geometrical phases are defined as [7]:

$$
\begin{aligned}
& \gamma_{N m}^{(\mathrm{d})}=-\frac{1}{\hbar} \int_{0}^{t}\langle\psi(\boldsymbol{r}, \tau)|H| \psi(r, \tau)\rangle \mathrm{d} \tau, \\
& \gamma_{N m}^{(\mathrm{g})}(t)=\int_{0}^{t}\left\langle\phi_{N m}(\tau)\left|\mathrm{i} \frac{\partial}{\partial \tau}\right| \phi_{N m}(\tau)\right\rangle \mathrm{d} \tau,
\end{aligned}
$$


where $\left|\phi_{N m}(t)\right\rangle$ represents the function $\phi_{N m}(r, \varphi, t)$ by the form of the Dirac note. The dynamical phase and geometrical phase may be computed directly from (46), but the process is a little complicated. Here we provide a simple method to get them.

First, $\phi_{N m}(R) \phi_{m}(\varphi)$ is the eigenfunction of a two-dimensional harmonic oscillator in cylindrical coordinate with the Hamiltonian

$$
H_{\xi \eta}=\frac{p_{\xi}^{2}+p_{\eta}^{2}}{2}+\frac{\xi^{2}+\eta^{2}}{2}
$$

where $R^{2}=\xi^{2}+\eta^{2}, \xi=x /(g \sqrt{\hbar}), \quad \eta=y /(g \sqrt{\hbar})$, and $p_{\xi}=-\mathrm{i} \hbar \partial / \partial \xi$, $p_{\eta}=-\mathrm{i} \hbar \partial / \partial \eta$. In rectangular coordinate, the Hamiltonian is the superposition of two one-dimensional harmonic oscillators with the coordinates $\xi$ and $\eta$. Writing the eigenstates of these two harmonic oscillators as $\psi_{n_{x}}(\xi)$ and $\psi_{n_{y}}(\eta)$ with $n_{x}=n_{y}=0,1,2, \ldots$, the functions $\psi_{n_{x}}(\xi) \psi_{n_{y}}(\eta)$ form a complete set. The function $\phi_{N m}(R) \phi_{m}(\varphi)$ can be expanded as

$$
\phi_{N m}(R) \phi_{m}(\varphi)=\sum_{n_{x} n_{y}} C_{n_{x} n_{y}} \psi_{n_{x}}(\xi) \psi_{n_{y}}(\eta)
$$

The normalization condition is $\sum_{n_{x} n_{y}}\left|C_{n_{x} n_{y}}\right|^{2}=1$ and the relation of the quantum number $N$ with $n_{x}, n_{y}$ is $N=n_{x}+n_{y}$. Substituting (48) into (44), we have

$$
\phi_{N m}(r, \varphi, t)=\sum_{n_{x} n_{y}} C_{n_{x} n_{y}} \psi_{n_{x}}(x, t) \psi_{n_{y}}(y, t)
$$

where

$$
\begin{aligned}
& \psi_{n_{x}}(x, t)=\psi_{n_{x}}(\xi) \exp \left(\frac{\mathrm{i} M \dot{g}}{2 \hbar g} x^{2}\right), \\
& \psi_{n_{y}}(y, t)=\psi_{n_{y}}(\eta) \exp \left(\frac{\mathrm{i} M \dot{g}}{2 \hbar g} y^{2}\right),
\end{aligned}
$$

each of which is the eigenfunction of the invariant for TOHO [5]. The expectation value for the Hamiltonian of TOHO is a special case of that in [10]:

$$
\left(j+\frac{1}{2}\right) \hbar\left\{\frac{1}{2 M}\left[\frac{1}{g^{2}}+(M \dot{g})^{2}\right]+\frac{1}{2} M \Omega^{2} g^{2}\right\}, \quad j=n_{x}, n_{y} .
$$

Now the dynamical phase (46) can be calculated without difficulty

$$
\begin{aligned}
\gamma_{N m}^{(\mathrm{d})} & =-\left(N+\frac{1}{2}\right)\left\{\frac{1}{2 M}\left[\frac{1}{g^{2}}+(M \dot{g})^{2}\right]+\frac{1}{2} M \Omega^{2} g^{2}\right\} \\
& -\frac{m}{2} \int_{0}^{t} \varpi_{c}(\tau) \mathrm{d} \tau .
\end{aligned}
$$

The geometrical phase of the wave function of TOHO can be obtained from [10]:

$$
\gamma_{j}^{(\mathrm{g})}(t)=-\left(j+\frac{1}{2}\right) \int_{0}^{t}\left[g^{2}(\tau) \frac{\mathrm{d}}{\mathrm{d} \tau}\left(\frac{M(\tau) \dot{g}(\tau)}{2 g(\tau)}\right)\right] \mathrm{d} \tau, \quad j=n_{x}, n_{y},
$$


the geometrical phase (46) can be calculated easily

$$
\gamma_{N m}^{(\mathrm{g})}(t)=-(N+1) \int_{0}^{t}\left[g^{2}(\tau) \frac{\mathrm{d}}{\mathrm{d} \tau}\left(\frac{M(\tau) \dot{g}(\tau)}{2 g(\tau)}\right)\right] \mathrm{d} \tau
$$

Using the auxiliary condition $(20)$, it is not difficult to prove $\gamma_{N m}^{(\mathrm{d})}(t)+$ $\gamma_{N m}^{(\mathrm{g})}(t)=\gamma_{N m}(t)$.

\section{The driven system}

For the driven system, for example the time-dependent driven TOHO

$$
\frac{\mathrm{d}}{\mathrm{d} t}(M \dot{q})+M \omega^{2} q=F(t)
$$

the equation of motion can be rewritten as

$$
\frac{\mathrm{d}}{\mathrm{d} t}\left[M \dot{q}-g_{0}(t)\right]+M \omega^{2}\left[q-f_{0}(t)\right]=0,
$$

where $g_{0}(t)=M \dot{f}_{0}(t)$ is the momentum corresponding to the displacement $f_{0}(t)$, which is a solution of the equation

$$
\frac{\mathrm{d}}{\mathrm{d} t}\left[M \dot{f}_{0}(t)\right]+M \omega^{2} f_{0}(t)=F(t)
$$

If making the transformation

$$
q \rightarrow q-f_{0}, \quad p=M \dot{q} \rightarrow p-g_{0},
$$

Eq. (3) will become (56) and at the same time the invariant (14) changes into

$$
Q=\left(\frac{q-f_{0}}{\rho}\right)^{2}+\left[\rho\left(p-g_{0}\right)-M \dot{\rho}\left(q-f_{0}\right)\right]^{2},
$$

which is the invariant of the driven system (55). Similar results can be found for TTHO, or TTHO in a magnetic field, or even the time-dependent driven generalized harmonic oscillator (TDGHO) [10]. (For the sake of space, we do not give the details here.)

The form (59) may be derived by similar calculations to that in Sec. 2. The only difference is that now we should set $q(t)-f_{0}(t)=A(t) / \cos f(t)$.

It was known that for a driven system, the wave function is a wave packet with the center moving according to the classical equation of motion [10]. The center motion induces new terms in the dynamical phase and geometrical phase. Taking TDGHO as an example, the driving induced dynamical phase and geometrical phase can be cast into the form

$$
\begin{aligned}
& \gamma^{(\mathrm{d})}(t)=-\frac{1}{\hbar} \int_{0}^{t} H_{\mathrm{cl}}(\tau) \mathrm{d} \tau, \quad \gamma^{(\mathrm{g})}(t)=-\frac{1}{\hbar} \int_{0}^{t} g_{0}(\tau) \mathrm{d} f_{0}(\tau)+\Delta(t)-\Delta(0) \\
& \Delta(t)=\frac{1}{\hbar}\left[f_{0}(t) g_{0}(t)+\left(\frac{M \dot{\rho}}{2 \rho}-\lambda M\right) f_{0}^{2}(t)\right]
\end{aligned}
$$


where $H_{\mathrm{cl}}$ is the classical Hamiltonian of TDGHO. From the momentum (11a) in [10], one knows that the driving induced term in the quantum Hamiltonian expectation value (21) in [10] is just the classical Hamiltonian of TDGHO, which yields the dynamical phase in (60) here. The first term in the geometrical phase in (60) is the phase of the de Broglie wave of the center motion and the rest is an elementary function. For a periodic evolution, $\Delta(T)-\Delta(0)=0$, the geometrical phase in (60) equals the phase of the matter wave associated with the center motion of the wave function.

\section{Conclusions}

From the exact solutions to the equations of motion, the invariants for TOHO and TTHO in a static magnetic field are derived. Utilizing the trial function method, the wave function of TTHO in a static magnetic field is easily achieved in cylindrical coordinate and the dynamical and geometrical phases are obtained by expressing the wave function as the superpositions of the wave functions of TTHO in rectangular coordinate. For the driven system, the physical meaning of the driving induced dynamical and geometrical phases is analyzed.

\section{Acknowledgments}

This project was supported by the LiuHui Fund for Applied Mathematics.

\section{References}

[1] H.R. Lewis, Phys. Rev. Lett. 18, 510 (1967); H.R. Lewis, W.B. Riesenfeld, J. Math. Phys. 10, 1458 (1969).

[2] P.G.L. Leach, SIAM J. Appl. Math. 34, 496 (1978).

[3] M. Lutzky, Phys. Lett. A 68, 3 (1978).

[4] M.F. Guasti, A. Gil-Villegas, Phys. Lett. A 292, 243 (2002).

[5] I.A. Pedrosa, Phys. Rev. A 55, 3219 (1997).

[6] C.A.S. Ferreira, P.T.S. Alencar, J.M.F. Bassalo, Phys. Rev. A 66, 024103 (2002).

[7] Y. Aharonov, J. Anandan, Phys. Rev. Lett. 58, 1593 (1987).

[8] S.S. Mizrahi, Phys. Lett. A 138, 465 (1989).

[9] K. Husimi, Prog. Theor. Phys. 9, 381 (1953).

[10] M.L. Liang, H.B. Wu, Phys. Scr. 68, 41 (2003).

[11] See for example Y.F. Liu, Y.A. Lei, J.Y. Zeng, Phys. Lett. A 231, 9 (1997). 